\title{
Genetic variation in $A K T 1$ is linked to dopamine-associated prefrontal cortical structure and function in humans
}

\author{
Hao-Yang Tan, Kristin K. Nicodemus, Qiang Chen, Zhen Li, Jennifer K. Brooke, \\ Robyn Honea, Bhaskar S. Kolachana, Richard E. Straub, Andreas Meyer-Lindenberg, \\ Yoshitasu Sei, Venkata S. Mattay, Joseph H. Callicott, and Daniel R. Weinberger \\ Clinical Brain Disorders Branch, Genes, Cognition and Psychosis Program, Division of Intramural Research Programs, \\ National Institute of Mental Health, NIH, Bethesda, Maryland, USA.
}

\begin{abstract}
AKT1-dependent molecular pathways control diverse aspects of cellular development and adaptation, including interactions with neuronal dopaminergic signaling. If AKT1 has an impact on dopaminergic signaling, then genetic variation in $A K T 1$ would be associated with brain phenotypes related to cortical dopaminergic function. Here, we provide evidence that a coding variation in $A K T 1$ that affects protein expression in human $B$ lymphoblasts influenced several brain measures related to dopaminergic function. Cognitive performance linked to frontostriatal circuitry, prefrontal physiology during executive function, and frontostriatal graymatter volume on MRI were altered in subjects with the $A K T 1$ variation. Moreover, on neuroimaging measures with a main effect of the $A K T 1$ genotype, there was significant epistasis with a functional polymorphism (Val158Met) in catechol-O-methyltransferase [COMT], a gene that indexes cortical synaptic dopamine. This genetic interaction was consistent with the putative role of $A K T 1$ in dopaminergic signaling. Supportive of an earlier tentative association of $A K T 1$ with schizophrenia, we also found that this $A K T 1$ variant was associated with risk for schizophrenia. These data implicate $A K T 1$ in modulating human prefrontal-striatal structure and function and suggest that the mechanism of this effect may be coupled to dopaminergic signaling and relevant to the expression of psychosis.
\end{abstract}

\begin{abstract}
Introduction
Dopaminergic abnormalities have long served as a major framework for understanding the pathophysiology as well as pharmacology of psychosis, such as schizophrenia, and also associated cognitive deficits, particularly those affecting executive function and working memory. Classically, D1 receptors, implicated in the maintenance of relevant information during the working memory delay period (1), couple through $G \alpha_{\text {s }}$ protein to stimulate the production of cAMP and the activity of PKA (2). Conversely, D2 receptors, which in neural models play critical roles marking salience, prediction errors, and updating and manipulating new information (3), couple through $\mathrm{G \alpha}_{\mathrm{i} / \mathrm{o}}$ protein to reduce cAMP production and PKA activity (2). Downstream from PKA, dopamine- (DA-) and cAMPregulated phosphoprotein of molecular weight 32 (DARPP-32) is a key signaling integrator that regulates an array of subsequent neurophysiological processes (2). Genetic variation in DARPP-32 has recently been found to have an impact on normal human variation in frontostriatal cognitive performance, neostriatal volume and physiologic activation, and functional connectivity between striatum and prefrontal cortex; DARPP-32 has also been implicated in risk for schizophrenia (4). The cAMP/PKA/DARPP-32 pathway, however, is not the only molecular network that transduces DA signals in dopaminoceptive neurons (2).
\end{abstract}

Nonstandard abbreviations used: BA, Brodmann area; COMT, catechol-O-methyltransferase; DA, dopamine; DARPP-32, DA- and cAMP-regulated phosphoprotein of molecular weight 32; fMRI, functional MRI; LD, linkage disequilibrium; WAIS-R, Wechsler Adult Intelligence Scale - Revised; WMS-R, Wechsler Memory Scale - Revised.

Conflict of interest: The authors have declared that no conflict of interest exists. Citation for this article: J. Clin. Invest. 118:2200-2208 (2008). doi:10.1172/JCI34725.
In addition to the canonical cAMP/PKA pathway, D2 receptors may also signal through an AKT1/GSK-3 signaling cascade via $\beta$-arrestin 2 (5). Of particular note, this AKT/GSK-3 pathway influences the expression of DA-associated psychomotor behaviors that, in transgenic models, have been predictably modulated by dopaminergic agonists and antagonists; this pathway also appears independent of the CAMP-associated one and represents what we believe is a novel means by which D2 receptor signaling and associated cognitive and neuropsychiatric effects could be mediated (5-7). AKT1-knockout mice, in particular, showed evidence of abnormal prepulse inhibition of startle (8) and poorer working memory performance under dopaminergic agonist challenge as well as concurrent changes in prefrontal pyramidal dendritic ultrastructure, possibly mediated by downstream alterations in the expression of genes controlling neuronal development in prefrontal cortex (7). On the other hand, typical and atypical antipsychotics, either directly or through 5-hydroxytryptamine $\left(5-\mathrm{HT}_{2 \mathrm{~A}}\right)$ receptors, enhanced AKT/GSK-3 signaling (9). AKT1 has also recently been found to indirectly have an impact on DA signaling by regulating the trafficking of presynaptic DA transporters, which remove DA from extracortical synapses (10).

Furthering the plausibility that these findings relate to the pathophysiology of psychosis, decreased AKT1 protein levels have been observed in lymphoblasts and postmortem prefrontal cortices of patients with schizophrenia $(8,11)$. This observation led to initial evidence of genetic association of $A K T 1$ with schizophrenia, a finding that has been reported in at least 4 studies in white populations $(8,11-13)$ and in at least 2 studies of other populations (14, $15)$. Some studies, however, have not found association between $A K T 1$ and schizophrenia $(16,17)$, and there have been inconsis- 
Table 1

AKT1 association with cognitive factors in healthy individuals from the Clinical Brain Disorders Branch sample

\begin{tabular}{|c|c|c|c|c|c|c|c|c|c|}
\hline $\begin{array}{l}\text { Factor } \\
\text { no. }\end{array}$ & Cognitive factor & $\begin{array}{l}\text { rs2494732 } \\
\text { (SNP5) }\end{array}$ & $\begin{array}{l}\text { rs1130233 } \\
\text { (SNP4) }\end{array}$ & $\begin{array}{c}\text { rs3730358 } \\
\text { (SNP3) }\end{array}$ & $\begin{array}{c}\text { rs1130214 } \\
\text { (SNP2) }\end{array}$ & $\begin{array}{l}\text { rs3803300 } \\
\text { (SNP1) }\end{array}$ & Change & $P$ & Global $P$ \\
\hline 1 & Verbal memory & & & & & & & & $>0.05$ \\
\hline 2 & Working memory & & & & & & & & $>0.05$ \\
\hline 3 & Visual memory & & & & & & & & $>0.05$ \\
\hline \multirow[t]{6}{*}{4} & IQ/processing speed & & & & & & & & $>0.05$ \\
\hline & & $\underline{G}$ & $\underline{G}$ & $\underline{C}$ & & & $\uparrow$ & 0.011 & $<0.0001^{\mathrm{B}}$ \\
\hline & & $\underline{\mathrm{G}}$ & $\underline{\bar{A}}$ & $\underline{\bar{C}}$ & & & $\downarrow$ & 0.0019 & \\
\hline & & & $\underline{\mathrm{G}}$ & $\underline{\bar{C}}$ & $\underline{T}$ & & $\uparrow$ & 0.017 & $0.031^{\mathrm{B}}$ \\
\hline & & & $\underline{\bar{A}}$ & $\underline{\bar{C}}$ & $\underline{\mathrm{G}}$ & & $\downarrow$ & 0.0014 & \\
\hline & & & $\overline{A^{C}}$ & & & & $\downarrow$ & 0.003 & \\
\hline 5 & Wisconsin Card Sort & & & & & & & & $>0.05$ \\
\hline 6 & Attention & & & & & & & & $>0.05$ \\
\hline \multirow[t]{2}{*}{7} & Digit span & & & & & & & & $>0.05$ \\
\hline & Coding strand & $\mathrm{A} / \mathrm{G}$ & $\mathrm{G} / \mathrm{A}$ & $\mathrm{C} / \mathrm{T}$ & $\mathrm{G} / \mathrm{T}$ & $\mathrm{G} / \mathrm{A}$ & & & \\
\hline
\end{tabular}

SNP designations in parentheses according to Emamian et al. (8). Arrows pointing up and down denote association with increased and decreased scores, respectively. Underlines denote 3-SNP sliding-window haplotypes. ${ }^{A}$ Designated rs2498799 in the earlier literature. ${ }^{B}$ Global $P$ value applies to both $3-S N P$ haplotypes shown. ${ }^{\mathrm{C}}$ Single SNP association. $n=319$.

tencies across studies in SNPs and alleles showing strongest association. Thus, the AKT1 association with schizophrenia per se has been tentative, although even if the genetic association were valid, inconsistency might not be surprising given the impact of phenotypic and genetic heterogeneity and the importance of gene-gene and gene-environment interactions (18).

An alternative strategy for characterizing the role of genes in complex human behaviors such as psychosis is to study genetic association at the level of brain structure and function. There is a growing body of data showing that genes weakly associated with complex constellations of behavioral symptoms are much more strongly associated with in vivo brain measures if indeed these measures reflect the specific neural processes more directly affected by the biology of the genes $(19,20)$. Given the preclinical data implicating AKT1 and dopaminergic frontostriatal function and cellular structure (5-8), we hypothesized that human genetic variation in AKT1 might be associated with phenotypes referable to the frontostriatal dopaminergic system. Specifically, we propose that even in healthy individuals without confounders of illness and previous psychotropic drug use, genetic variation in AKT1 might robustly affect cognitive brain phenotypes such as DA-dependent executive cognition and corresponding MRI measures of prefrontal physiology and cortical-striatal morphology. To determine further the potential role of DA signaling in these genetic associations and because DA is critical for the physiologic tuning of prefrontal cortical circuits (21), we also predicted that the imaging phenotypes would evidence epistatic interactions between AKT1 and a gene that indexes cortical synaptic DA bioavailability, catechol-O-methyltransferase (COMT) $(22,23)$. The functional COMT Val158Met polymorphism has become part of a useful strategy for studying the effects of synaptic DA levels in cortex. COMT Val alleles have relatively increased enzyme activity (22) and thus reduced cortical synaptic DA (23) and increased cortical disinhibition of mesencephalic-striatal DA $(24,25)$, which manifest as deficits in prefrontal neural signal-to-noise tuning efficiency when assayed with functional MRI (fMRI) (25-30) and with EEG (31). Thus, if the mechanism of $A K T 1$ acts at least in part via a link to dopaminergic signaling in prefrontal cortex, then relatively deleterious COMT genotypes and relatively deleterious $A K T 1$ genotypes should interact epistatically and manifest as nonadditively greater abnormalities in these same prefrontal cortical circuits. Finally, we also examined AKT1 for genetic association with schizophrenia in a case-control and nonindependent family-based sample.

\section{Results}

We studied AKT1 effects on human subjects at several phenotypic levels -319 individuals in terms of cognitive tests, a subset of 32 individuals in terms of AKT1 protein expression in lymphoblasts, independent subsets of 46 and 68 individuals in terms of fMRI of brain, and an overlapping set of 171 individuals for structural brain MRI. We also examined AKT1 association with schizophrenia using the combined sample of 370 healthy individuals and 358 families having an offspring with schizophrenia. We engaged a conservative strategy to test specific hypotheses based on a priori evidence from prior findings in the literature and to thus limit multiple testing. First, we searched within 3-SNP sliding-window haplotypes for association with cognition across the 5 common AKT1 SNPs originally studied by Emamian et al. as well as in subsequent schizophrenia association studies $(8,11,12,14-16)$. Cognition was indexed by a set of 7 factors derived from a battery of neuropsychological tests that have identified cognitive deficits related to increased genetic risk for schizophrenia (32-34). Only SNPs contained in globally significant haplotypes were individually examined. The potential functional relevance of the single SNP showing strong cognitive association was then further interrogated in the lymphocyte expression data and in the multimodal neuroimaging data under strict statistical control for the number of independent brain-imaging elements ("voxels") tested. Finally, we tested for genetic association with schizophrenia in all 5 SNPs in the set of 358 affected families and in 370 unrelated healthy subjects. Exploratory secondary analyses were also performed in this clinical dataset on an additional 7 SNPs to survey potential common haplotypes not accounted for by the SNPs selected from the earlier literature (Supplemental Table 1; supplemental material 

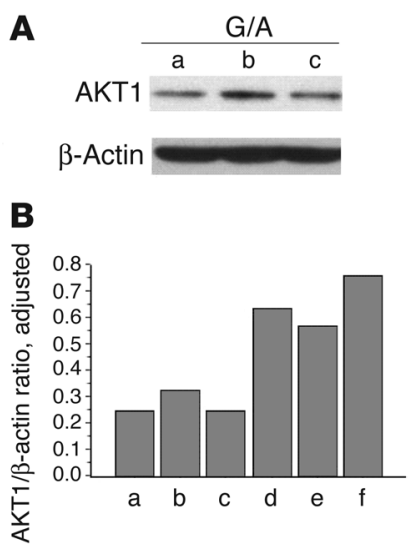

\section{Figure 1}

AKT1 expression in human lymphoblasts. (A) Representative Western blot images of AKT1 and $\beta$-actin protein bands from lymphoblasts of 3 individuals with $A K T 1 \mathrm{rs} 1130233 \mathrm{G} / \mathrm{A}$ and 3 individuals with $\mathrm{G} / \mathrm{G}$. (B) AKT1/ $\beta$-actin ratio of the same individuals. (C) AKT1/ $\beta$-actin ratio across all 13 individuals with $\mathrm{G} / \mathrm{A}$ and 19 individuals with $\mathrm{G} / \mathrm{G}$ genotype showing a significant genotype effect $(P<0.05)$. Error bars are \pm 1 SEM.

available online with this article; doi:10.1172/JCI34725DS1). Association with cognition was also tested in these additional SNPs, and the results, which were consistent with the primary analyses reported here, are presented in the supplementary material (Supplemental Table 2). We also genotyped the COMTVal158Met SNP (22) to examine for the hypothesized AKT1/DA signaling relationship in brain circuitry measured by neuroimaging. All SNPs were in Hardy-Weinberg equilibrium (Fisher's exact test; $P>0.05$ ), and each had minor allele frequencies of more than $5 \%$. In healthy individuals, the overall linkage disequilibrium (LD) across the AKT1 SNPs spanning a block defined by rs2498804 and rs3730358 was moderate to strong (Supplemental Figure 1). There was no correlation of COMT and AKT1 SNP alleles in the sample of normal subjects tested for interaction of these genes.

Neurocognitive phenotypes. We first examined 3-SNP sliding-window haplotypes across the 5 main AKT1 SNPs from the literature. Haplotype associations with each of the 7 cognitive factors (33) in our normal sample were determined using a score-test-based method (35) from which global and haplotype-specific test statistics and $P$ values were derived. Global significance was used to screen haplotypic association across each of these cognitive domains (Table 1). Factor 4, comprising measures of IQ, executive function, and processing speed from subtests of the Wechsler Adult Intelligence Scale - Revised (WAIS-R), the Trail Making Test, and the Verbal and Category Fluency Test (33), was associated with significant $A K T 1$ haplotypic variation, the strongest comprising SNPs rs3730358, rs1130233 (formerly aliased rs2498799 in the earlier literature), and rs2494732 (global $P<10^{-6}$, uncorrected). The specific GAC haplotype was associated with reduced factor 4 scores $(P<0.002$, uncorrected, or $P<0.02$, Bonferroni corrected for the 8 possible haplotype combinations in these 3 SNPs). Of the individual SNPs in this haplotype, a linear regression analysis revealed that the rs1130233 A allele was associated with reduced factor 4 scores $(P=0.003$, uncorrected, or $P<0.01$, Bonferroni corrected for the 3 SNPs tested against cognitive factor 4$)$. The neuropsychological measures comprising this cognitive factor reflect IQ, processing speed, and executive function engaging brain networks that include frontal cortex and striatum (36-39).

Functional impact of AKT1 genetic variation on gene expression in vivo. Based on the positive association of rs1130233 with cognition in our normal sample, particularly with IQ, executive function, and processing speed - engaging frontostriatal processes linked with cortical DA function and implicated in schizophrenia $(32,40,41)$ - we determined the effect of this synonymous coding SNP on AKT1 protein in peripheral lymphoblasts derived from a subset of the healthy subjects used in our cognitive and imaging association analyses $(n=32$; Supplemental Table 3$)$. rs1130233 has also been implicated in a number of overlapping haplotypes previously associated with risk for schizophrenia, though the allele implicated in the risk haplotypes has been inconsistent $(8,11-14)$. That this same SNP is functional or monitors another functional variant is suggested by an earlier study showing an association of its minor A allele with reduced AKT1 protein levels and increased apoptosis following irradiation stress in human lymphoblasts (42). In the present study, we confirmed that individuals who are carriers of the A allele had significantly reduced AKT1 immunoreactivity on Western blot compared with major allele homozygote individuals ( $\mathrm{t}_{30}=2.14, P=0.041$; Figure 1 ).

Impact of AKT1 genetic variation on brain function. We then investigated the impact of rs1130233 genotype on brain function using fMRI in 2 independent samples of healthy subjects. Prefrontal function was assayed using the 2-back task - a robust paradigm engaging prefrontal executive working memory processes that have been linked to IQ $(38,39)$ and cortical DA function $(25,26$, 43-45). The first fMRI dataset $(n=46$ healthy subjects; Supplemental Table 3) comprised $25 \mathrm{G} / \mathrm{G}$ homozygote individuals and 21 individuals who were minor allele (A) carriers (including $3 \mathrm{~A}$ homozygotes). These subjects were also selected to be homozygous for alleles at COMT Val158Met (24 Met and $22 \mathrm{Val}$ ) to increase functional variability in the sample for the subsequent effort to examine the interaction effects of these 2 genes. There were no differences in sex, age, education, IQ, and importantly, 2-back performance indices across the AKT1 or COMT groups $(P>0.1$; Supplemental Table 4). Thus, since the genotype groups did not differ in task performance, differences in the fMRI data would reflect differences in how the information is processed in the brain, i.e., the efficiency of engagement of the cortical networks that handle the information and not task performance per se (28). Within a contiguous prefrontal region of interest defined by the main effect of 2-back task activation $(P<0.05$, corrected for false-discovery rate; Supplemental Table 5), the minor allele of SNP rs1130233, associated previously with reduced performance on cognitive tests and reduced AKT1 protein levels, engaged increased prefrontal activation, which suggests a relatively inefficient activation phenotype (Brodmann area [BA] 9: Montreal Neurological Institute coordinates $-38,30,36 \mathrm{~mm}, \mathrm{z}$ score $(z)=3.47 ; P=0.0003$, uncorrected, or $P<0.05$, small-volume corrected according to the principles of Gaussian random fields [ref. 46]; Figure 2A). This phenotype, i.e., the relative "inefficiency" of cortical engagement during the $\mathrm{N}$-back task, defined as the increased magnitude of the response in the context of fixed task performance, has been linked to reduced cortical DA function in normal subjects $(26,28,30,47)$ and in patients with Parkinson disease $(48,49)$.

To confirm this result, we performed a similar analysis on an independent sample of healthy volunteers comprising 37 AKT1 rs1130233 G/G individuals and 31 A carriers (including 4 A homo- 

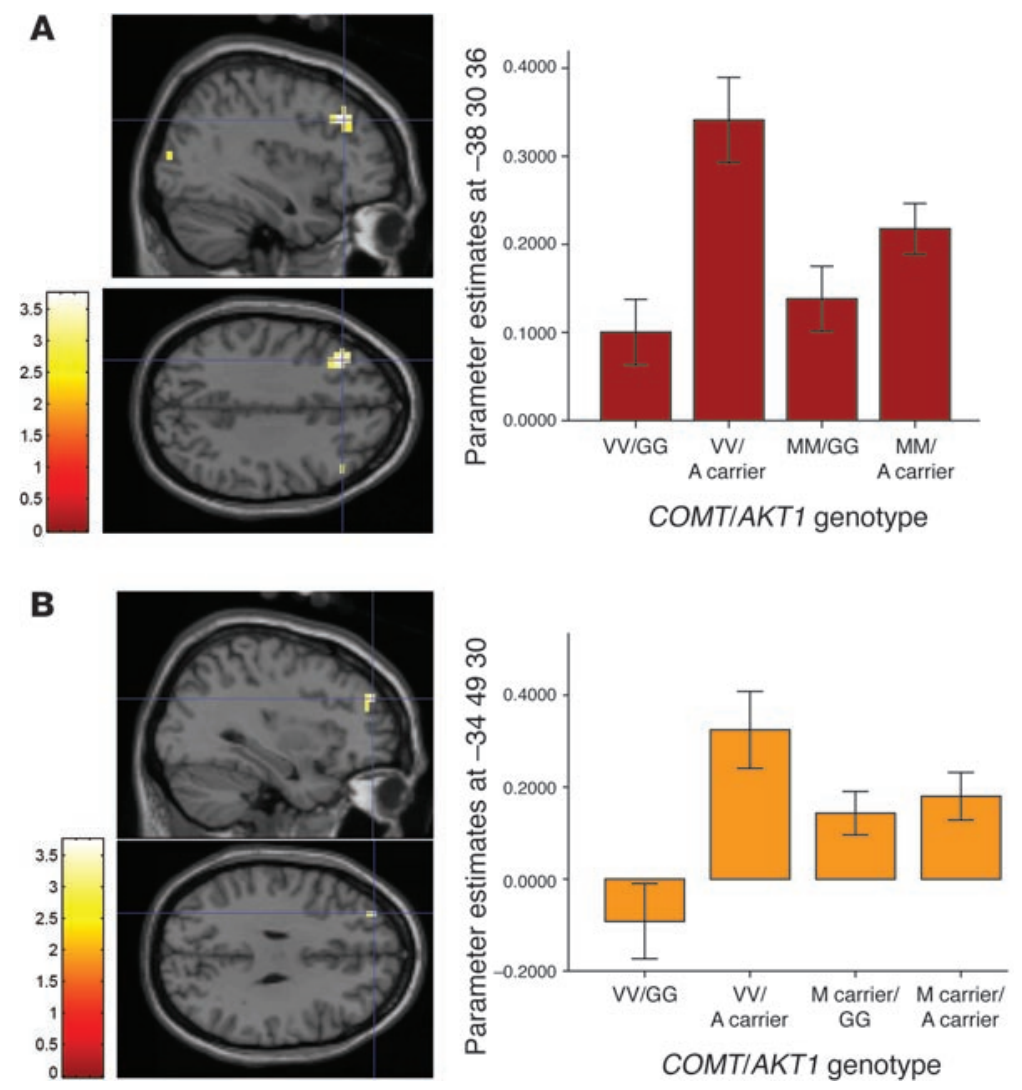

\section{Figure 2}

Functional neuroimaging. (A) The overlayed functional brain image shows the main effect of 2-back task-related activation in AKT1 rs1130233 minor allele carriers versus major allele homozygotes at the dorsolateral prefrontal cortex in the first dataset $(P<0.005$, uncorrected for display only; $n=46$ ). The graph shows the corresponding extracted parameter estimates representing task-related activation according to $C O M T$ and $A K T 1$ genotype. There was an $A K T 1$-by-COMT interaction at $P<0.05$ (see Results) where combined deleterious COMT Val homozygotes and AKT1 A-carriers had disproportionately inefficient activation. (B) These effects were confirmed in the second dataset $(n=68)$. The overlayed functional brain image was displayed at $P<0.005$, uncorrected; the corresponding extracted parameter estimates showed a COMT-by-AKT1 interaction effect at $P<0.05$. See Results for details. Error bars are \pm 1 SEM zygotes; $n=68$ healthy subjects). In this sample not selected for COMT Val158Met genotypes, there were 55 who were Met carriers and 13 who were Val homozygotes. Again, there were no differences in sex, age, education, IQ, or 2-back performance indices across $A K T 1$ or COMT groups $(P>0.1$; Supplemental Table 4$)$. Within a 20-mm bounding box of prefrontal 2-back activation centered at the previous activation peak (BA 9: coordinates $-38,30,36 \mathrm{~mm}$ ), the AKT1 SNP rs1130233 A allele was associated with a similar pattern of inefficient activation (BA 9/10: coordinates $-34,49,30$ $\mathrm{mm}, z=2.76, P<0.05$, small-volume corrected, or $P=0.003$, uncorrected; Figure $2 \mathrm{~B}$ ). Exploratory analyses also revealed another prefrontal region of $A K T 1$-associated inefficient activation (BA 47: coordinates $41,19,-12 \mathrm{~mm}, z=3.03, P<0.001$, uncorrected; Supplemental Figure 2).

Impact of AKT1 genetic variation on brain structure. Because AKT1 is implicated in several key neurodevelopmental pathways and neuronal morphology (7), we predicted that genetic variation having an impact on brain function would affect brain measures of structural development. This may be independent of AKT1's putative role in DA signaling, but it may also reflect at least in part the effects of DA on brain development that have been described $(7,50-52)$. Changes in gray-matter volume relative to $A K T 1$ variation were explored using optimized voxel-based morphometry in an overlapping sample of 171 healthy subjects (103 AKT1 rs $1130233 \mathrm{G} / \mathrm{G}$ individuals and $68 \mathrm{~A}$ carriers including $8 \mathrm{~A}$ homozygotes; 126 COMT Met carriers and 45 Val homozygotes; Supplemental Table 3). Here, the AKT1 SNP rs1130233 minor allele was associated with relatively reduced gray-matter volumes in the bilateral caudate (coordinates $-10,7,3 \mathrm{~mm}, z=3.79$,
$P<0.05$, small-volume corrected; coordinates $12,7,4 \mathrm{~mm}$, $z=3.37, P<0.05$, small-volume corrected) and right prefrontal cortex (BA 47: 37, 18, $-12 \mathrm{~mm}, z=3.73, P<0.05$, small-volume corrected; Figure 3 and Supplemental Table 6).

Epistasis between AKT1 and COMT on brain function and structure. To examine the predicted coupling between dopaminergic signaling and $A K T 1$ at the level of in vivo prefrontal function and structure $(5,7,8)$, we tested COMT-AKT1 interaction effects on orthogonal parameter estimates extracted from only the peak prefrontal regions showing the AKT1 rs1130233 main effects noted above. All 3 neuroimaging samples yielded significant epistatic effects on these parameters. Extracted parameter estimates from the peak at BA 9 (coordinates $-38,30,36 \mathrm{~mm}$ ) in the first fMRI dataset showed a significant $A K T 1$-by-COMT interaction: individuals with $A K T 1$ minor alleles and COMT Val homozygote genotypes engaged disproportionately (i.e., nonadditive) "inefficient" activation [via 2-by-2 factorial ANOVA across the 2 genes each with 2 genotype levels, with $\mathrm{F}(1,42)=4.466, P=0.041$; Figure $2 \mathrm{~A}$ and Supplemental Figure $3 \mathrm{~A}]$. This was subsequently replicated in the second dataset at BA $9 / 10$ [coordinates $-34,49,30 \mathrm{~mm} ; \mathrm{F}(1,64)=5.703, P=0.020$; Figure $2 \mathrm{~B}$ and Supplemental Figure $3 \mathrm{~B}]$, with similar findings at BA 47 [coordinates 41, 19, -12; F(1,64) = 7.251, $P=0.009$; Supplemental Figures 2 and $3 \mathrm{C}$ ]. Finally, in terms of brain structure, extracted gray-matter volume estimates from the right prefrontal cortex (BA 47: coordinates $37,18,-12 \mathrm{~mm}$ ) also showed an AKT1-by-COMT interaction $[\mathrm{F}(1,162)=10.16, P=0.002]$ in that individuals with combined deleterious AKT1 minor and COMT Val alleles had disproportionately reduced gray-matter volume at this location (Figure 3B and Supplemental Figure 3D). 

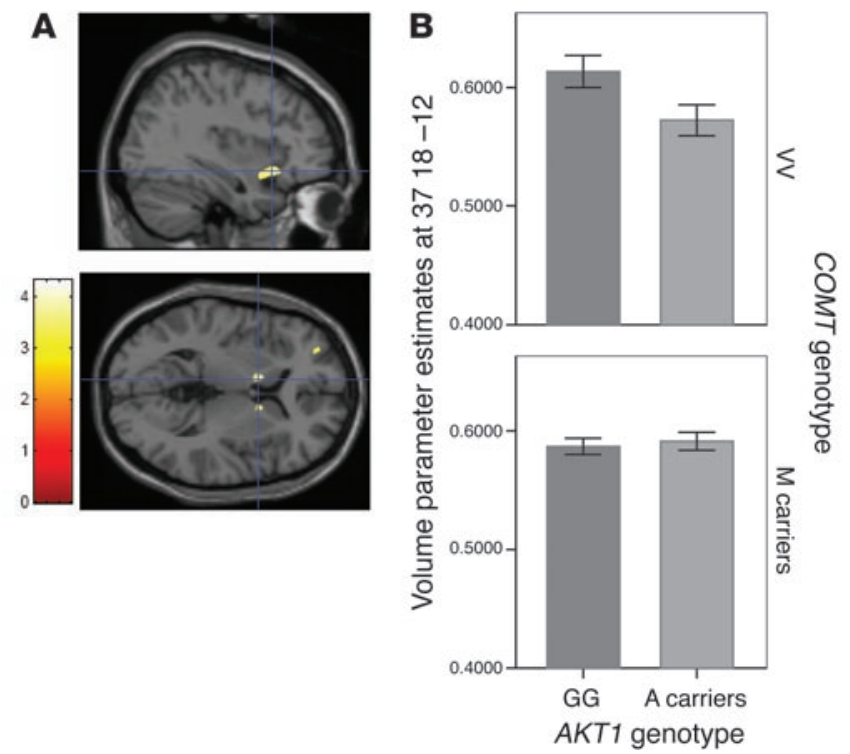

Figure 3

Structural neuroimaging. (A) Main effects of AKT1 SNP rs1130233 on gray-matter volumes showing reduced volume of minor allele carriers versus major allele homozygotes at the ventral prefrontal cortex (top left panel) and bilateral caudate (bottom left panel). Images were thresholded at $P<0.001$, uncorrected, for display $(n=171$ healthy subjects). (B) Extracted volume parameter estimates showed an $A K T 1$-by-COMT interaction in the prefrontal cortex at $P<0.005$ (see Results), where combined deleterious COMT Val homozygotes and $A K T 1 \mathrm{~A}$ allele carriers had disproportionately reduced gray-matter volume. Error bars are \pm 1 SEM.

AKT1 association with schizophrenia. We performed a family-based (358 probands/families) and a nonindependent case-control (358 probands, 370 controls) association study using the Clinical Brain Disorders Branch sample. A logistic regression analysis in the casecontrol dataset controlling for sex and age suggested that the A genotype at rs1130233 was associated with increased risk for schizophrenia (odds ratio 2.50, 95\% CI, 1.14-5.50; $P=0.024$ ). This SNP, however, was not significantly associated in the nonindependent family-based association study. None of the other SNPs showed association $(P>0.1)$ in either dataset. Thus, while our results are tentative with respect to association with risk for schizophrenia, rs1130233 has previously been implicated in risk for schizophrenia across overlapping haplotypes with variable intermarker LD (8, 11-14), so the prior probability of association with this SNP might be considered as a possible adjustment in our statistics (53).

\section{Discussion}

We have taken a strategy of genetic association of $A K T 1$ with brain phenotypes related to DA function to examine in humans a potential effect of AKT1 on DA-mediated cellular function implicated in basic studies. In examining the effect of a genetic variant in AKT1 that had an impact on the expression of AKT1 protein in lymphoblasts, we found that this SNP influenced, even in healthy individuals, frontostriatal cognitive tasks taxing processing speed, IQ, and executive cognitive control-cardinal DA-mediated cognitive functions (36-40, 54, 55); this SNP also influenced "tuning" of a prefron- tal physiological phenotype previously linked to cortical DA function $(26,28,30,49)$. Moreover, this same genotype predicted reduced gray-matter volume in parts of the frontostriatal network. Prefrontal regions showing AKT1 main effects in $\mathrm{AMRI}$ and MRI volumetry further evidenced epistasis with COMT, a gene involved in regulating cortical synaptic DA levels $(22,23)$. These results provide converging evidence implicating measurable AKT1 gene effects on human prefrontal structure and function, consistent with preclinical evidence that couples AKT1 to dopaminergic signaling and downstream effects on prefrontal cellular structure and animal models of cognition $(5,7,8,10)$. Our data also suggest that the mechanisms of prior associations of $A K T 1$ with psychosis, a condition associated with DA abnormalities in brain $(56,57)$ and treated with antidopaminergic drugs, could involve these biologic processes.

At the level of neurocognition, $A K T 1$ genetic variation mapped onto cognitive factors representing tasks engaging IQ, processing speed, and executive cognitive control processes (Table 1). Specifically, we found strong association in normal subjects with scores on cognitive factor 4 , which consists of WAIS-R measures of arithmetic, similarities and picture completion, and processing speed measures of digit-symbol substitution, trail making, and letter and category fluency. These and related cognitive measures have been implicated in brain circuitry inclusive of frontal and striatal networks (36-39, $41,54,58,59)$. Dopaminergic signaling has long been implicated in executive cognition and processing speed and has recently been shown to be especially important in selecting, updating, and stabilizing new information during initial encoding and in the rapid shifting of cognitive sets $(3,30,60)$. Executive cognition and processing speed involve DA-dependent loops connecting the prefrontal cortex and striatum (55), also implicated in our neuroimaging results. AKT1 genetic variation did not map strongly onto other cognitive factors engaging episodic memory or attention nor factors potentially representing other aspects of executive function (e.g., factors 2 and 5). It is conceivable that the residual variance loaded onto factors 2 and 5 represents executive or decision processes with less emphasis for speed or processes like simple maintenance or retrieval of information. To the limited extent these standard behavioral tests and factor analyses were able to parse the array of executive cognitive processes (36), these latter processes appeared less strongly related to $A K T 1$ genetic variation. On the other hand, given recent evidence that tasks taxing more complex aspects of executive processing speed strongly represent cognitive impairment in schizophrenia and in relatives of patients with schizophrenia $(32,41,61-63)$, it is plausible that factor 4 represented such a combination of processes selectively impaired in relation to genetic risk for schizophrenia. Thus, the association of $A K T 1$ genetic variation to the quantitative traits representing these aspects of IQ, executive cognition, and processing speed, strongest at SNP rs1130233, is consistent with the proposed coupling between AKT1 and D2-receptor signaling (5, 7, $8,10)$, the latter implicated in neural models of selective gating and rapid updating of information critical in executive cognition engaging prefrontal-striatal neural systems $(3,64)$.

rs1130233 has previously been implicated in AKT1 expression levels and in mediating radiation-induced apoptosis of lymphocytes (42). We confirmed its effect on the expression of AKT1 protein in lymphoblasts from a subgroup of the normal subjects in our sample and confirmed the directionality of the allelic association. Consistent with basic models suggesting deficits in AKT phosphorylation and signaling underlying cognitive deficits $(5,7$, 8), the A allele at rs1130233 associated with poorer cognitive func- 
tion and relative deficits in neuroimaging measures correspondingly evidenced reduced levels of AKT1 in the lymphoblasts of a subset of the same individuals (Figure 1). Thus, within the constraints of biological differences in the expression of physiological function between cell types, lymphoblasts potentially provide a useful model to test the functional status of genetic variation in the same living human subjects evaluated with neurocognitive tests and neuroimaging (65). This is exemplified here by genetically mediated differences in AKT1 protein abundance, assumed to reflect similar differences at the level of brain.

The N-back fMRI paradigm has been a robust tool for engaging prefrontal networks during executive cognition. This paradigm also provides a reliable quantitative measure of the physiological efficiency trait; absent task performance as a confounder, the increased activation and physiological inefficiency phenotype has been consistently associated with schizophrenia and with increased genetic risk for schizophrenia $(20,66,67)$. This measure has also been shown to vary predictably with cortical dopaminergic tuning in an increasing number of pharmacological and gene-based human studies $(4,25-29,47,68,69)$, including in patients with Parkinson disease $(48,49)$. The results of these imaging studies are consistent with fundamental signal-to-noise tuning characteristics of cortical neurons based on the action of DA $(1,21)$. Hence, the prefrontal physiological efficiency trait measured by this fMRI paradigm closely reflects at least in part fundamental DA-dependent processes and related genetic control mechanisms. In this context, it is remarkable that human genetic variation in AKT1 associated with its reduced expression also predictably had an impact on in vivo prefrontal physiological inefficiency, consistent with DA-dependent deficits in working memory in $A K T$-knockout models, as well as the role AKT plays in dopaminergic signaling in neurons $(5,7,8)$. Furthermore, it is intriguing that this proposed intimate DA-AKT relationship was mapped onto prefrontal physiological efficiency during executive cognition in an interaction between $A K T 1$ genetic variation and cortical synaptic DA bioavailability indexed by the COMT Val158Met polymorphism $(22,23)$. In particular, the finding of epistatically increased prefrontal inefficiency in individuals having genetic variants associated with both reduced AKT1 expression and reduced cortical DA is consistent with the notion that these deleterious effects, when combined, should result in a disproportionately detuned state during DAsensitive executive cognition.

It might also be expected that genetic variation in AKT1 and resultant changes in functional protein expression have an impact on neural structure. Arguably, our structural MRI findings relate to this and may further bridge the translation across animal models, $A K T 1$ gene expression, and human prefrontal executive cognition. As noted, Lai et al. demonstrated that AKT-knockout mice had working memory behavioral deficits and evidenced parallel changes in prefrontal pyramidal dendritic ultrastructure and downstream alterations in the expression of genes controlling synaptic function, neuronal development, myelination, and actin polymerization in prefrontal cortex (7). Chronic opiate exposure also has been shown to downregulate AKT signaling and lead to decreased DA cell size in ventral tegmental area (51). While MRI volumetry does not directly measure specific neuronal ultrastructure, the AKT1 association with gray-matter volume is strengthened by its location at the precise frontostriatal networks implicated in DA-mediated executive cognition. Together, our findings of reduced gray-matter volumes in these dopaminergic brain networks in individuals bearing the $A K T 1$ allele for inefficient prefrontal physiological function and reduced $A K T 1$ expression is consistent with the possibility that this genetic variant had an impact on both neural architecture and function.

Further support for the conclusion that these structural effects of $A K T 1$ are modulated at least in part by dopaminergic signaling comes from our finding that the same prefrontal region showing AKT1 structural effects evidenced an interaction with COMT Val$158 \mathrm{Met}$. While the precise mechanisms through which reduced cortical DA and/or reciprocal disinhibited mesencephalic-striatal DA in COMTVal homozygotes $(24,25,55)$ potentiates the prefrontal structural changes related to $A K T 1$ variation is unclear in this systems-level analysis, their cooccurrence in a statistical epistasis supports the hypothesized relationship between the DA-AKT1 system and their impact on regional prefrontal ultrastructure demonstrated in murine models $(7,51)$. It is interesting to note that similar ultrastructural changes have been described in DA-depleted animals (70), which would be analogous to the COMT Val allele being associated with reduced synaptic DA $(22,23)$. These structural findings thus provide another level of validation to the primary effect of AKT1 on DA-dependent human prefrontal physiology.

We have performed a complex set of experiments addressing association of multiple variants in a gene with many phenotypic measures. Thus, the potential for spurious association because of multiple testing is important to consider. Our strategy has been to rationally integrate a number of related phenotypic measures in a hypothesis-driven approach, with confirmation in independent samples and conservative statistical correction for the number of tests performed. In the primary analysis on cognitive phenotypes, a limited number of cognitive factors were derived from the neuropsychological battery upon which association with 3-SNP haplotypes from the 5 original SNPs in the literature (8) was examined and statistically corrected to implicate the factor representing aspects of processing speed, executive function, and IQ. Only 1 SNP most strongly implicated in our cognition data was subsequently examined in the lymphoblast and neuroimaging datasets. The functional imaging datasets were controlled for behavioral performance (and other demographic) confounders and statistically controlled for the limited number of prefrontal voxels tested based on the main effect of a well-validated DA-dependent working memory paradigm, and the findings were then independently replicated. Similarly stringent control for the number of brain voxels tested was employed for the structural imaging findings. Critically, we explicitly investigated DA-dependent prefrontal processes in terms of epistasis between the target AKT1 SNP and COMT Val/Met at the prefrontal peak showing the orthogonal AKT1 main effect. This interaction effect occurred in all 3 neuroimaging datasets, thus providing converging and compelling evidence that $A K T 1$ genetic variation has an impact on DA-dependent prefrontal systems biology in living humans.

Finally, in the context of prior evidence implicating $A K T 1$ in risk for schizophrenia $(8,11-14)$ combined with an association found here in our case-control study, our data further illustrate that a modest signal at the level of complex behavior shows much stronger association with a series of biologic intermediate phenotypes linked to the effects of $A K T 1$ in brain. We suggest that AKT1 SNP rs1130233 or other variants in LD with this SNP potentially mediate schizophrenia risk through reduced AKT1 signaling in concert with putative dopaminergic processes, which then have an impact on prefrontal-striatal structure and prefrontal function during exec- 
utive cognition. Despite this apparent convergence of data linking AKT1 to brain biology, the evidence that rs1130233 is a causative SNP mediating association with schizophrenia is limited, as prior association of this single SNP with schizophrenia has been modest and differing risk haplotypes containing this SNP in different populations have been implicated $(8,11-14)$. Thus, rs1130233, which is in variable LD with the other SNPs in haplotypes previously showing some association with schizophrenia, is likely linked to multiple risk haplotypes across samples, for which it gets "credit" as a marker, even as the alleles or haplotypes have defied exact replication (53, 71). In spite of the uncertainty of association of $A K T 1$ with schizophrenia, our results support notions that genetic mechanisms modestly associated with clinical psychiatric syndromes are more powerfully examined through effects at the level of fundamental brain mechanisms and cognitive processes, particularly those based on prior basic science evidence (20). Of note, modulating the AKTrelated pathways in the direction of that apparently observed in psychosis is a major therapeutic goal for cancer (72). This not only adds to the interesting epidemiological observation that risk for schizophrenia and cancer might be inversely related (73) but should also encourage further preclinical and human studies specifying these pathways, implicated also in neurodegeneration (74), in the search for more effective therapies that target differentiated effects specific to each of these important diseases.

\section{Methods}

Subjects. Research subjects were ascertained as part of the Clinical Brain Disorders Branch Sibling Study (32). For genetic association studies in families, we examined families with a proband with schizophrenia $(n=358)$. A nonindependent case-control study comprised 358 unrelated probands and 370 unrelated healthy controls. Subjects were all of European ancestry to minimize genetic heterogeneity and stratification artifacts. All subjects were from 18 to 60 years of age, were above 70 in IQ, and gave written informed consent. Exclusion criteria were significant medical problems, history of loss of consciousness for greater than 5 minutes, alcohol or drug abuse/dependence within the last 12 months, and electroconvulsive therapy within the last 6 months. All subjects were interviewed by a research psychiatrist using the structured interview for DSM-IV. For probands, data from psychiatric records were also evaluated during diagnostic ascertainment. The study was approved by the National Institute of Mental Health Institutional Review Board.

DNA collection and genotyping. DNA was extracted from whole blood using standard procedures. All genotypes were determined using the $5^{\prime}$ exonuclease TaqMan assay; SNP probe and primer sets were acquired as Assays on Demand from Applied Biosystems. We genotyped the 5 original AKT1 SNPs in the literature (8) for the primary analyses reported here. We also selected 7 additional SNPs from National Center for Biotechnology Information databases based on position and allele frequencies. (These latter results are reported in the supplementary material as they were similar to that in the primary analyses.) Genotype accuracy was assessed by regenotyping within a subsample, and reproducibility was routinely greater than $99 \%$. Genotyping completion rate was greater than $95 \%$, and genotyping errors were identified within the family dataset through non-Mendelizing transmissions and haplotype inconsistency via MERLIN version 1.0.1 (http://www.sph.umich.edu/csg/abecasis/Merlin/). We measured LD between markers with the $\mathrm{D}^{\prime}$ and $\mathrm{r}^{2}$ statistics from unrelated case and control haplotypes with the program LDMAX within the Graphical Overview of Linkage Disequilibrium (GOLD) software package (75).

Cognitive testing. The neurocognitive battery comprised neuropsychological tests with evidence of heritability and association with risk for schizophrenia (32-34). It included the Wechsler Memory Scale - Revised (WMS-R), WAIS-R (arithmetic, similarities, digit-symbol substitution, and picture completion), Trail Making Test parts A and B, the Verbal and Category Fluency Test, the Continuous Performance Test, the N-back task, the California Verbal Learning Test, the Judgment of Line Orientation, and the Wisconsin Card Sorting Test. These 24 subtests were reducible via principal components and confirmatory factor analyses to a 7-factor solution (33). These factors were more independent than the individual subtest scores and putatively closer to the individuals' underlying psychometric structure than any single subtest (33). Factor 1 was loaded on verbal episodic memory measures from the WMS-R and California Verbal Learning Test; factor 2 on aspects of working memory from the N-back task; factor 3 on spatial episodic memory measures from WMS-R and Judgement of Line Orientation; factor 4 on IQ, executive cognitive control, and processing speed measures from WAIS-R, trails A and B, and letter and category fluency; factor 5 on logical reasoning measures from the Wisconsin Card Sorting Test; factor 6 on attentional measures from the Continuous Performance Test; and factor 7 on measures from the WMS-R digit span backwards and forwards (33). AKT1 genetic contribution to these cognitive factors was analyzed in a group of 319 healthy individuals for whom neuropsychological data was available. Three-SNP sliding window haplotype analyses were performed using the score-test-based statistic implemented in the R package haplo.stats (35). Individual SNP analyses of implicated haplotypes were subsequently performed via a linear regression model to identify variation associated with cognitive factor scores.

AKT1 protein expression in lymphocytes. Epstein-Barr virus-transformed B lymphoblasts from a subgroup of 32 healthy controls (19 individuals with $A K T 1$ SNP rs $1130233 \mathrm{G} / \mathrm{G}$ and 13 individuals with A alleles) were prepared and AKT1 protein levels were analyzed by immunoblot (65). In brief, proteins extracted in buffer containing $250 \mathrm{mM}$ Tris- $\mathrm{HCl}$ ( $\mathrm{pH} 7.4$ ), $100 \mathrm{mM} \mathrm{NaCl}, 1 \%$ Triton X-100, 0.1\% SDS, 0.5\% deoxycholic acid, $1 \mathrm{mM}$ $\mathrm{p}$-nitrophenylguanidinobenzoate, and protease inhibitor cocktails I and II (Sigma-Aldrich) were separated using SDS-PAGE on 4\%-12\% Tris-Glycine gel (Invitrogen). After separation, the proteins were transferred to a polyvinylidene difluoride membrane (Millipore) and probed with antibodies to AKT1 (07-416; Millipore) or $\beta$-actin (A5441; Sigma-Aldrich) on a polyvinylidene difluoride membrane (Millipore). Horseradish peroxidase-conjugated anti-rabbit or mouse IgG antibody (Pierce Biotechnology) was used to detect the primary antibodies. Chemiluminescence detection was performed using enhanced chemoluminescence (GE Healthcare) or Super pico signal (Pierce Biotechnology). The protein bands within the linear range of the standard curve were imaged, and the relative optical density of each band was measured and analyzed using NIH Image software. Between-group comparison of relative protein levels across the AKT1 genotypes was then analyzed using $t$ tests.

Functional neuroimaging of executive cognition. To examine in vivo neural circuitry, blood oxygen level-dependent (BOLD) fMRI were acquired from 2 independent groups of 46 and 68 healthy subjects as they performed the 2 -back task. This task engages executive working memory processes (44) and consisted of continual presentation of visual stimuli in which every number was both a probe and a target (i.e., 100\% target). The numbers 1-4 appeared randomly every 1.8 seconds for $500 \mathrm{~ms}$ at set locations at the points of a diamond-shaped box. Subjects were to recall the stimulus seen in "N" trials previously by means of a fiber-optic response box with buttons arrayed in the same configuration as the stimuli presented on the screen. Thirty-second epochs of 0-back alternated with 2-back.

Whole-brain BOLD fMRI data were collected on a 3-T scanner (GE Systems) with a GE-EPI pulse sequence acquisition of 24 contiguous slices (echo time: $30 \mathrm{~ms}$; repetition time: 2 seconds; flip angle: $90^{\circ}$; field of view: $24 \mathrm{~cm}$; matrix: $64 \times 64$; voxel dimensions: $3.75 \times 3.75 \times 6 \mathrm{~mm}$ ). All $\mathrm{fMRI}$ data 
were preprocessed and spatially normalized to a common stereotaxic space (Montreal Neurological Institute template) with SPM99 software (Wellcome Department of Imaging Neuroscience; http://www.fil.ion.ucl.ac.uk/spm) and individually tested for motion artifacts as described previously (44).

Single-subject contrast images were then entered into a second level of analysis with subject as a random factor. We examined the effect of working memory (2-back vs. 0-back) in the sample. This task robustly engaged the prefrontal cortex, where activation had been observed to reliably reflect processing efficiency dependent on cortical DA in an inverted U-shaped curve $(25,28)$. Given the hypothesis that AKT1 is coupled to cortical dopaminergic effects, we restricted our analyses of AKT1 gene effects in the first fMRI sample to prefrontal cortical regions activated by the task at $P<0.05$, corrected for false-discovery rate (76). AKT1 SNP rs1130233 A carriers versus $\mathrm{G}$ homozygote effects were subsequently interrogated within this smaller search volume and statistically corrected according to the theory of Gaussian random fields (46) at $P<0.05$. To determine the reliability of this finding, we searched in the second fMRI sample within a 20 -mm bounding box centered on the peak showing AKT1 effects in the first sample, using a similar corrected threshold within this smaller volume. Results at $P<0.001$, uncorrected, were also reported. Epistatic interaction between $A K T 1$ and COMT effects was subsequently extracted from significant peaks with orthogonal AKT1 effects and examined using ANOVA at $P<0.05$ in this higher-level analysis.

Structural neuroimaging. To examine brain structure, MRI images from 171 healthy subjects were acquired in a $1.5 \mathrm{~T}$ GE scanner using a T1-weighted spoiled grass sequence (repetition time: $24 \mathrm{~s}$; echo-time: $5 \mathrm{~s}$; field-of-view: $24 \mathrm{~cm}$; matrix: $256 \times 256$ voxels; flip-angle: $\left.45^{\circ}\right)$ with 124 sagittal slices $(0.94 \times 0.94 \times 1.5 \mathrm{~mm}$ resolution $)$. These images were processed and analyzed using optimized voxel-based morphometry with customized templates in SPM2 as previously described (77). Resulting gray-matter images were smoothed with a $12-\mathrm{mm}$ Gaussian kernel before statistical analysis in
SPM2 using a generalized linear model. The effects of AKT1 SNP rs1130233 genotypes $G / G$ versus A carriers were examined using an analysis of covariance model adjusted for the orthogonalized first and second polynomials of age, sex, WAIS-IQ, and total gray-matter volume, as previously described (77). We reported peaks at $P<0.001$, uncorrected, and also applied $P<0.05$ correction for false discovery rate $(76)$ in prefrontal-striatal regions of interest defined using the Wake Forest University PickAtlas (http://www. fmri.wfubmc.edu/download.htm). Epistatic interaction between $A K T 1$ and COMT effects were extracted and examined as in the fMRI data.

AKT1 genetic association with schizophrenia. We examined AKT1 genetic association for schizophrenia using a case-control dataset from the Clinical Brain Disorders Branch comprising 358 unrelated patients with schizophrenia and 370 healthy controls, all of European ancestry. Single AKT1 SNPs were analyzed using logistic regression, controlling for sex and age. A nonindependent family study comprising available siblings and parents of these 358 patients was analyzed using FBAT for single SNPs and 3-SNP sliding window haplotypes with permutation testing for significance assessment (FBAT; http://www.biostat.harvard.edu/ fbat/fbat.htm).

\section{Acknowledgments}

This work was supported by the National Institute of Mental Health Intramural Research Program. We thank Amanda Law and Bai Lu for critique of the manuscript.

Received for publication December 11, 2007, and accepted in revised form April 2, 2008.

Address correspondence to: Daniel R. Weinberger, NIMH, NIH, 10 Center Drive, Room 4S-235, Bethesda, Maryland 20892, USA. Phone: (301) 402-7564; Fax: (301) 480-7795; E-mail: weinberd@ mail.nih.gov.
1. Williams, G.V., and Goldman-Rakic, P.S. 1995. Modulation of memory fields by dopamine $\mathrm{D} 1$ receptors in prefrontal cortex. Nature. 376:572-575.

2. Greengard, P. 2001. The neurobiology of slow synaptic transmission. Science. 294:1024-1030.

3. O'Reilly, R.C. 2006. Biologically based computational models of high-level cognition. Science. 314:91-94.

4. Meyer-Lindenberg, A., et al. 2007. Genetic evidence implicating DARPP-32 in human frontostriatal structure, function, and cognition. J. Clin. Invest. 117:672-682.

5. Beaulieu,J.M., et al. 2005. An Akt/ $\beta$-arrestin 2/PP2A signaling complex mediates dopaminergic neurotransmission and behavior. Cell. 122:261-273.

6. Beaulieu, J.M., et al. 2004. Lithium antagonizes dopamine-dependent behaviors mediated by an AKT/glycogen synthase kinase 3 signaling cascade. Proc. Natl. Acad. Sci. U. S. A. 101:5099-5104.

7. Lai, W.S., et al. 2006. Akt1 deficiency affects neuronal morphology and predisposes to abnormalities in prefrontal cortex functioning. Proc. Natl. Acad. Sci. U. S. A. 103:16906-16911.

8. Emamian, E.S., Hall, D., Birnbarum, M.J., Karayiogou, M., and Gogos, J.A. 2004. Convergent evidence for impaired AKT1-GSK3b signaling in schizophrenia. Nat. Genet. 36:131-137.

9. Alimohamad, H., Rajakumar, N., Seah, Y.-H., and Rushlow, W. 2005. Antipsychotics alter the protein expression levels of [beta]-catenin and GSK-3 in the rat medial prefrontal cortex and striatum. Biol. Psychiatry. 57:533-542.

10. Wei, Y., et al. 2007. Dopamine transporter activity mediates amphetamine-induced inhibition of Akt through a Ca2+/Calmodulin-Dependent Kinase II-Dependent Mechanism. Mol. Pharmacol. 71:835-842.
11. Thiselton, D.L., et al. 2007. AKT1 is associated with schizophrenia across multiple symptom dimensions in the Irish study of high density schizophrenia families. Biol. Psychiatry. 63:449-457.

12. Schwab, S.G., et al. 2005. Further evidence for association of variants in the AKT1 gene with schizophrenia in a sample of European sib-pair families. Biol. Psychiatry. 58:446-450.

13. Norton, N., et al. 2007. Association analysis of AKT1 and schizophrenia in a UK case control sample. Schizophr. Res. 93:58-65.

14. Bajestan, S.N., et al. 2006. Association of AKT1 haplotype with the risk of schizophrenia in Iranian population. Am.J. Med. Genet. B Neuropsychiatr. Genet. 141:383-386.

15. Ikeda, M., et al. 2004. Association of AKT1 with schizophrenia confirmed in a Japanese population. Biol. Psychiatry. 56:698-700.

16. Ide, M., et al. 2006. Failure to support a genetic contribution of AKT1 polymorphisms and altered AKT signaling in schizophrenia. J. Neurochem. 99:277.

17. Liu, Y.L., et al. 2005. Absence of significant associations between four AKT1 SNP markers and schizophrenia in the Taiwanese population. Psychiatr. Genet. 16:39-41.

18. Straub, R.E., and Weinberger, D.R. 2006. Schizophrenia genes - famine to feast. Biol. Psychiatry. 60:81.

19. Meyer-Lindenberg, A.S., and Weinberger, D.R. 2006. Intermediate phenotypes and genetic mechanisms of psychiatric disorders. Nat. Rev. Neurosci. 7:818-827.

20. Weinberger, D.R., et al. 2001. Prefrontal neurons and the genetics of schizophrenia. Biol. Psychiatry. 50:825-844.

21. Vijayraghavan, S., Wang, M., Birnbaum, S.G., Williams, G.V., and Arnsten, A.F.T. 2007. Inverted-U dopamine D1 receptor actions on prefrontal neurons engaged in working memory. Nat. Neurosci. 10:376-384.

22. Chen, J., et al. 2004. Functional analysis of genetic variation in catechol-O-methyltransferase (COMT): effects on mRNA, protein, and enzyme activity in postmortem human brain. Am. J. Hum. Genet. 75:807-821.

23. Yavich, L., Forsberg, M.M., Karayiorgou, M., Gogos, J.A., and Mannisto, P.T. 2007. Site-specific role of catechol-O-methyltransferase in dopamine overflow within prefrontal cortex and dorsal striatum. J. Neurosci. 27:10196-10209.

24. Akil, M., et al. 2003. Catechol-O-methyltransferase genotype and dopamine regulation in the human brain. J. Neurosci. 23:2008-2013.

25. Meyer-Lindenberg, A., et al. 2005. Midbrain dopamine and prefrontal function in humans: interaction and modulation by COMT genotype. Nat. Neurosci. 8:594-596.

26. Egan, M.F., et al. 2001. Effect of COMT Val108/158 Met genotype on frontal lobe function and risk for schizophrenia. Proc. Natl. Acad. Sci.U. S. A. 98:6917-6922

27. Gothelf, D., et al. 2005. COMT genotype predicts longitudinal cognitive decline and psychosis in $22 \mathrm{q} 11.2$ deletion syndrome. Nat. Neurosci. 8:1500-1502.

28. Mattay, V.S., et al. 2003. Catechol O-methyltransferase val158-met genotype and individual variation in the brain response to amphetamine. Proc. Natl. Acad. Sci. U. S. A. 100:6186-6191.

29. McIntosh, A.M., et al. 2007. Relationship of catechol-O-methyltransferase variants to brain structure and function in a population at high risk of psychosis. Biol. Psychiatry. 61:1127.

30. Tan, H.Y., et al. 2007. Catechol-O-methyltransferase Val158Met modulation of prefrontal-parietal-stria- 
tal brian systems during arithmetic and temporal transformations in working memory. J. Neurosci. 27:13393-13401.

31. Winterer, G., et al. 2006. Prefrontal electrophysiologic "noise" and catechol-O-methyltransferase genotype in schizophrenia. Biol. Psychiatry. 60:578-584.

32. Egan, M.F., et al. 2001. Relative risk for cognitive impairments in siblings of patients with schizophrenia. Biol. Psychiatry. 50:98-107.

33. Genderson, M.R., et al. 2007. Factor analysis of neurocognitive tests in a large sample of schizophrenic probands, their siblings, and healthy controls. Schizophr. Res. 94:231-239.

34. Cannon, T.D., et al. 2000. The inheritance of neuropsychological dysfunction in twins discordant for schizophrenia. Am. J. Hum. Genet. 67:369-382.

35. Schaid, D.J., Rowland, C.M., Tines, D.E., Jacobson, R.M., and Poland, G.A. 2002. Score tests for association between traits and haplotypes when linkage phase is ambiguous. Am. J. Hum. Genet. 70:425-434

36. Royall, D.R., et al. 2002. Executive control function: a review of its promise and challenges for clinical research. A report from the Committee on Research of the American Neuropsychiatric Association. J. Neuropsychiatry Clin. Neurosci. 14:377-405

37. Duncan, J., et al. 2000. A neural basis for general intelligence. Science. 289:457-460.

38. Gray, J.R., Chabris, C.F., and Braver, T.S. 2003. Neural mechanisms of general fluid intelligence. Nat. Neurosci. 6:316.

39. Kane, M.J., and Engle, R.W. 2002. The role of prefrontal cortex in working-memory capacity, executive attention, and general fluid intelligence: An individual-differences perspective. Psychon. Bull. Rev. 9:637.

40. Pantelis, C., et al. 1997. Frontal-striatal cognitive deficits in patients with chronic schizophrenia. Brain. 120:1823-1843.

41. Dickinson, D., Ramsey, M.E., and Gold, J.M. 2007 Overlooking the obvious: a meta-analytic comparison of digit symbol coding tasks and other cognitive measures in schizophrenia. Arch. Gen. Psychiatry. 64:532-542.

42. Harris, S.L., et al. 2005. Detection of functional single-nucleotide polymorphisms that affect apoptosis. Proc. Natl. Acad. Sci. U. S. A. 102:16297-16302.

43. Wang, M., Vijayraghavan, S., and Goldman-Rakic, P.S. 2004. Selective D2 receptor actions on the functional circuitry of working memory. Science. 303:853-856.

44. Callicott, J.H., et al. 2003. Complexity of prefrontal cortical dysfunction in schizophrenia: more than up or down. Am. J. Psychiatry. 160:2209-2215.

45. Aalto, S., Bruck, A., Laine, M., Nagren, K., and Rinne, J.O. 2005. Frontal and temporal dopamine release during working memory and attention tasks in healthy humans: a positron emission tomography study using the high-affinity dopamine D2 receptor ligand [11C]FLB 457. J. Neurosci.
25:2471-2477.

46. Worsley, K.J., et al. 1996. A unified statistical approach for determining significant signals in images of cerebral activation. Hum. Brain Mapp. 4:58-73.

47. Apud, J.A., et al. 2007. Tolcapone improves cognition and cortical information processing in normal human subjects. Neuropsychopharmacology. 32:1011-1020

48. Williams-Gray, C.H., Hampshire, A., Robbins, T.W., Owen, A.M., and Barker, R.A. 2007. Catechol Omethyltransferase val158met genotype influences frontoparietal activity during planning in patients with Parkinson's disease. J. Neurosci. 27:4832-4838.

49. Mattay, V.S., et al. 2002. Dopaminergic modulation of cortical function in patients with Parkinson's disease. Ann. Neurol. 51:156-164.

50. Beaulieu, J.-M., Gainetdinov, R.R., and Caron, M.G. 2007. The Akt-GSK-3 signaling cascade in the actions of dopamine. Trends Pharmacol. Sci. 28:166.

51. Russo, S.J., et al. 2007. IRS2-Akt pathway in midbrain dopamine neurons regulates behavioral and cellular responses to opiates. Nat. Neurosci. 10:93-99.

52. Levitt, P., Harvey, J.A., Friedman, E., Simansky, K., and Murphy, E.H. 1997. New evidence for neurotransmitter influences on brain development. Trends Neurosci. 20:269.

53. Clarke, G.M., Carter, K.W., Palmer, L.J., Morris, A.P., and Cardon, L.R. 2007. Fine mapping versus replication in whole-genome association studies. Am.J. Hum. Genet. 81:995-1005.

54. Salthouse, T.A. 1996. The processing-speed theory of adult age differences in cognition. Psychol. Rev. 103:403-428.

55. Alexander, G.E., DeLong, M.R., and Strick, P.L. 1986. Parallel organization of functionally segregated circuits linking basal ganglia and cortex. Annu. Rev. Neurosci. 9:357-381.

56. Laruelle, M., Kegeles, L.S., and Abi-Dargham, A. 2003. Glutamate, dopamine, and schizophrenia: from pathophysiology to treatment. Ann. N. Y. Acad. Sci. 1003:138-158.

57. Winterer, G., and Weinberger, D.R. 2004. Genes, dopamine and cortical signal-to-noise ratio in schizophrenia. Trends Neurosci. 27:683-690.

58. Bäckman, L., et al. 2000. Age-related cognitive deficits mediated by changes in the striatal dopamine system. Am. J. Psychiatry. 157:635-637.

59. Gray, J.R., and Thompson, P.M. 2004. Neurobiology of intelligence: science and ethics. Nat. Rev. Neurosci. 5:471.

60. Seamans, J.K., and Yang, C.R. 2004. The principal features and mechanisms of dopamine modulation in the prefrontal cortex. Prog. Neurobiol. 74:1-57.

61. Tan, H.Y., Choo, W.C., Fones, C.S.L., and Chee, M.W.L. 2005. fMRI study of maintenance and manipulation processes within working memory in first-episode schizophrenia. Am. J. Psychiatry. 162:1849-1858.

62. Silver, H., Feldman, P., Bilker, W.B., and Gur, R.C. 2003. Working memory deficit as a core neuropsychological dysfunction in schizophrenia. Am.J.
Psychiatry. 160:1809-1816

63. Barch, D.M., et al. 2001. Selective deficits in prefrontal cortex function in medication-naive patients with schizophrenia. Arch. Gen. Psychiatry. 58:280-288.

64. Gruber, A.J., Dayan, P., Gutkin, B.S., and Solla, S.A. 2006. Dopamine modulation in the basal ganglia locks the gate to working memory. J. Comput. Neurosci. 20:153-166.

65. Sei, Y., et al. 2007. Neuregulin1-induced cell migration is impaired in schizophrenia: association with neuregulin 1 and catechol-o-methyltransferase gene polymorphisms. Mol. Psychiatry. 12:946-957.

66. Callicott, J.H., et al. 2003. Abnormal fMRI response of the dorsolateral prefrontal cortex in cognitively intact siblings of patients with schizophrenia. Am. J. Psychiatry. 160:709-719.

67. Glahn, D.C., et al. 2005. Beyond hypofrontality: a quantitative meta-analysis of functional neuroimaging studies of working memory in schizophrenia. Hum. Brain Mapp. 25:60-69.

68. Bertolino, A., et al. 2006. Additive effects of genetic variation in dopamine regulating genes on working memory cortical activity in human brain. J. Neurosci. 26:3918-3922.

69. Tan, H.Y., et al. 2007. Epistasis between catecholO-methyltransferase and type II metabotropic glutamate receptor 3 genes in working memory brain function. Proc. Natl. Acad. Sci. U. S. A. 104:12536-12541.

70. Sherren, N., and Pappas, B.A. 2005. Selective acetylcholine and dopamine lesions in neonatal rats produce distinct patterns of cortical dendritic atrophy in adulthood. Neuroscience. 136:445-456.

71. Lin, P.-I., Vance, J.M., Pericak-Vance, M.A., and Martin, E.R. 2007. No gene is an island: the flip-flop phenomenon. Am. J. Hum. Genet. 80:531-538.

72. Hennessy, B.T., Smith, D.L., Ram, P.T., Lu, Y., and Mills, G.B. 2005. Exploiting the PI3K-AKT pathway for cancer drug discovery. Nat. Rev. Drug Discov. 4:988-1004.

73. Levav, I., et al. 2007. Cancer risk among parents and siblings of patients with schizophrenia. $\mathrm{Br} . \mathrm{J}$. Psychiatry. 190:156-161.

74. Burke, R.E. 2007. Inhibition of mitogen-activated protein kinase and stimulation of Akt kinase signaling pathways: Two approaches with therapeutic potential in the treatment of neurodegenerative disease. Pharmacol. Ther. 114:261.

75. Abecasis, G.R., and Cookson, W.O. 2000. GOLD - graphical overview of linkage disequilibrium. Bioinformatics. 16:182-183.

76. Genovese, C.R., Lazar, N.A., and Nichols, T. 2002. Thresholding of statistical maps in functional imaging using the false discovery rate. Neuroimage. 15:870-878.

77. Pezawas, L., et al. 2004. The Brain-derived neurotrophic factor val66met polymorphism and variation in human cortical morphology. J. Neurosci. 24:10099-10102. 